\title{
ASPHERICITY OF SYMMETRIC PRESENTATIONS
}

\author{
Fulvia Spaggiari
}

Abstract

Using the notion of relative presentation due to Bogley and Pride, we give a new proof of a theorem of Prishchepov on the asphericity of certain symmetric presentations of groups. Then we obtain further results and applications to topology of low-dimensional manifolds.

\section{Relative presentations}

This section is devoted to recall some definitions and results on the asphericity of relative presentations according to [2].

A relative presentation is a triple $P=\langle H, X: R\rangle$ such that:

- $H$ is a group,

- $X=\left\{x_{1}, x_{2}, \ldots\right\}$ is a set of elements,

- $R$ is a set of words in the alphabet $H \cup X \cup X^{-1}$ of the form

$$
x_{1}^{\epsilon_{1}} h_{1} x_{2}^{\epsilon_{2}} h_{2} \cdots x_{n}^{\epsilon_{n}} h_{n}
$$

where $x_{i} \in X, \epsilon_{i}= \pm 1$ and $h_{i} \in H$.

We always assume that $R$ contains no proper powers, and that the words are cyclically reduced in the following sense: if $h_{i}=1$ and $x_{i}=$ $x_{i+1}$ (subscripts mod $n$ ), then $\epsilon_{i}=\epsilon_{i+1}$. The elements of $X \cup X^{-1}$ are also called $X$-symbols. Let $F(X)$ denote the free group on the set $X$. Then the group $G(P)$ defined by the relative presentation $P$ is the quotient of the free product $H * F(X)$ by the normal closure of $R$.

Let $R^{*}$ be the set of all cyclic permutations of words from $R \cup R^{-1}$ which begin with $X$-symbols. Let us consider the bar operator - on $R^{*}$ defined as follows. For any word $w \in R^{*}$, we write it in the form $w=u h$, where $h \in H$ and $u$ begins and ends with $X$-symbols. Then we set $\bar{w}=u^{-1} h^{-1} \in R^{*}$. Note that $\overline{\bar{w}}=w$, and $\bar{w}=w$ if and only if $w$ has the form $u h_{1} u^{-1} h_{2}$, where $u$ begins and ends with $X$-symbols and $h_{1}, h_{2}$ are elements of order 2 in $H$. The relative presentation $P=\langle H, X: R\rangle$ is

2000 Mathematics Subject Classification. 20F05, 20E22, 57M07, 57N13.

Key words. Relative presentations, labelled pictures, dipoles, asphericity, symmetric presentations, manifolds. 
slender if $\{w\}^{*} \cap R=\{w\}$, for any $w \in R$. The relative presentation $P$ is orientable if it is slender and no element of $R$ has a cyclic permutation fixed under the bar operator (i.e., no element of $R$ is a cyclic permutation of its inverse).

A picture $\mathbf{P}$ is a finite collection of pairwise disjoint discs $\left\{\Delta_{1}, \ldots, \Delta_{m}\right\}$ in the interior of a disc $D^{2}$, together with a finite collection of pairwise disjoint simple arcs $\left\{\alpha_{1}, \ldots, \alpha_{n}\right\}$ properly embedded in the closure of $D^{2} \backslash \bigcup_{i=1}^{m} \Delta_{i}$. For any $i=1, \ldots, m$, the corners of $\Delta_{i}$ are the closures of the connected components of $\partial \Delta_{i} \backslash \bigcup_{j=1}^{n} \alpha_{j}$. The regions of $\mathbf{P}$ are the closures of the connected components of $D^{2} \backslash\left(\bigcup_{i=1}^{m} \Delta_{i} \cup \bigcup_{j=1}^{n} \alpha_{j}\right)$. An inner region of $\mathbf{P}$ is a simply connected region of $\mathbf{P}$ which does not meet $\partial D^{2}$. The picture $\mathbf{P}$ is connected if $\bigcup_{i=1}^{m} \Delta_{i} \cup \bigcup_{j=1}^{n} \alpha_{j}$ is connected, and is spherical if $m \geq 1$ and $\left(\bigcup_{j=1}^{n} \alpha_{j}\right) \cap \partial D^{2}=\emptyset$.

A picture $\mathbf{P}$ is said to be labelled if:

- Each arc is equipped with a normal orientation, indicated by a short arrow meeting the arc transversely, and labelled by an $X$-symbol.

- Each corner of $\mathbf{P}$ is oriented anticlockwise with respect to the disk $\Delta_{i}$ in whose boundary it is contained, and labelled by an element of the group $H$.

Let $c$ be a corner of a disc $\Delta_{i}$ in the labelled picture $\mathbf{P}$. Then we denote by $w(c)$ the word obtained by reading in anticlockwise order the labels on the arcs and corners meeting $\partial \Delta_{i}$ beginning with the label on the arc which follows $c$. A label $x$ on an arc gives the generator $x$ or $x^{-1}$ if its normal orientation agrees or not with the reading sense.

A connected spherical labelled picture $\mathbf{P}$ is said to be a picture over the relative presentation $P=\langle H, X: R\rangle$ if the following conditions are satisfied:

- For any corner $c$ of $\mathbf{P}$, the word $w(c)$ belongs to $R^{*}$.

- If $h_{1}, h_{2}, \ldots, h_{\gamma(i)}$ is the sequence of the corner labels encountered in a clockwise traversal of the boundary of an inner region of $\mathbf{P}$, then $h_{1} h_{2} \cdots h_{\gamma(i)}=1$ in $H$.

Remark. An ordinary group presentation can be considered as the particular case of a relative presentation $P=\langle H, X: R\rangle$ for which $H=1$ (hence, there are no labels at corners of a picture over $P$ ).

A dipole in a picture $\mathbf{P}$ over a relative presentation $P$ consists of a pair of corners $c$ and $c^{\prime}$ with an arc $\alpha$ connecting the beginning of one corner with the end of the other such that $c$ and $c^{\prime}$ belong to the same region of $\mathbf{P}$ and $w\left(c^{\prime}\right)=\overline{w(c)}$. 
A relative presentation $P$ is said to be (combinatorially) aspherical if every nonempty connected spherical picture $\mathbf{P}$ over $P$ contains a dipole.

To complete the section, we illustrate a connection between the notion of aspherical relative presentation and the concept of topological asphericity.

Let $P=\langle H, X: R\rangle$ be a relative presentation for a group $G$. If $K(H, 1)$ is a Eilenberg-MacLane space for the group $H$, then consider the wedge

$$
K^{\prime}=K(H, 1) \vee\left(\vee_{x \in X} \mathbb{S}_{x}^{1}\right)
$$

For each $w \in R$, let $\phi_{w}: \mathbb{S}_{w}^{1} \rightarrow K^{\prime}$ be an attaching map which represents the word $w \in H * F(X) \cong \pi_{1}\left(K^{\prime}\right)$. Then the canonical complex $K(P)$ associated to $P$ is the $\mathrm{CW}$-complex

$$
K(P)=K^{\prime} \cup\left(\bigcup_{w \in R} D_{w}^{2}\right)
$$

where $D_{w}^{2}$ is a 2-cell attached to $K^{\prime}$ via $\phi_{w}$. By construction, we have the isomorphism $G \cong \pi_{1}(K(P))$.

Theorem 1. If $P=\langle H, X: R\rangle$ is an orientable (combinatorially) aspherical relative presentation for a group $G$, then the canonical complex $K(P)$ is topologically aspherical, that is, $K(P)=K(G, 1)$.

\section{A family of symmetric presentations}

Prishchepov [17] considered a family of symmetric presentations of groups depending on a finite number of positive integers:

$$
\begin{aligned}
P(r, n, k, s, q) & =\left\langle x_{1}, \ldots, x_{n}: \prod_{j=1}^{r} x_{i+(j-1) q}\right. \\
& \left.=\prod_{j=1}^{s} x_{i+k-1+(j-1) q} \quad(i=1, \ldots, n)\right\rangle
\end{aligned}
$$

where the subscripts are taken modulo $n, r \geq 2$, and $1 \leq q<n$. He gave arithmetic conditions on the parameters $(r, n, k, s, q)$ which imply the asphericity of the presentations $P(r, n, k, s, q)$ (see Section 3). Further results on the groups defined by these presentations and their generalizations can be found in [8].

The family $P(r, n, k, s, q)$ is very interesting from a topological point of view, and contains many classes of symmetric presentations, previously considered by several authors. 
- The presentations $P(r, n, r+1,1,1)$ define the Fibonacci groups $F(r, n), r \geq 2$ and $n \geq 3$ (see for example [14]). The group $F(2,2 m)$, $m \geq 2$, is the fundamental group of the $m$-fold cyclic covering of the 3-sphere branched over the figure-eight knot, as proved in [11]. The groups $F(n-1, n), n \geq 3$, are fundamental groups of Seifert fibered 3-manifolds [5].

- The presentations $P(r, n, 2, r-1,2)$ define the generalized Sieradski groups $S(r, n), r \geq 2, n \geq 2$, introduced and geometrically studied in [6]. The group $S(r, n)$ is the fundamental group of the $n$-fold cyclic covering of the 3 -sphere branched over the torus knot of type $(2 r-1,2)$, as shown in the quoted paper.

- The presentations $P(r, n, k+r, 1,1)$ and $P(r, n, r+1, s, 1)$ define the groups $F(r, n, k)$ and $H(r, n, k)$, respectively, for any $r \geq 2$, $n \geq 3$, and $k, s \geq 1$. These groups were introduced in [4] as natural generalizations of the Fibonacci groups $F(r, n)$. A lot of topological and algebraic results on these classes of groups can be found in the quoted paper and in [18].

- The presentations $P(2, n, 2,1, t)$ define the groups $H(n, t)$ studied in $[\mathbf{1 6}]$ and $[\mathbf{1 0}]$. The group $H(n, t)$ has infinite abelianization if and only if $n \equiv 0(\bmod 6)$ and $t \equiv 2(\bmod 6)$. The group $H(n, t)$ is perfect if and only if either $t=1$ or $n$ is coprime to 6 and $t \equiv 2(\bmod 6)$.

The following theorem, due to Gilbert and Howie, gives arithmetic conditions for the asphericity of groups $H(n, t)$.

Theorem 2. Suppose that $(n, t) \notin\{(8,3),(9,4),(9,7)\}$. Then the group $H(n, t)$ is aspherical, except for the values of $(n, t)$ listed below:

(1) $(n, 0)$, for $n \geq 2$,

(2) $(n, 2)$, for $n \geq 3$,

(3) $(n, n-1)$, for $n \geq 3$,

(4) $(2 t-1, t)$, for $t \geq 3$,

(5) $(2 t-2, t)$, for $t \geq 3$, and

(6) $(n, t)=(6,3),(7,3),(7,5),(9,3)$, or $(9,6)$.

- The presentations $P(2, n, k+1,1, m)$ define the groups $G_{n}(m, k)$, introduced in [7], and successively studied in [1]. They are natural generalizations of the Gilbert-Howie groups as $G_{n}(m, 1)=$ $H(n, m)$. The group $G_{n}(m, k)$ is said to be strongly irreducible if the parameters satisfy the following conditions: $0<m<k<n$, $\operatorname{gcd}(n, m, k)=1, \operatorname{gcd}(n, k)>1$, and $\operatorname{gcd}(n, k-m)>1$; otherwise, $G_{n}(m, k)$ is proved to be cyclic, a non-trivial free product, or a Gilbert-Howie group. 
The following theorem, due to Bardakov and Vesnin, gives arithmetic conditions for the asphericity of strongly irreducible groups $G_{n}(m, k)$.

Theorem 3. Let $G_{n}(m, k)$ be a strongly irreducible group. Then $G_{n}(m, k)$ is aspherical if all the following conditions are not satisfied:

(1) There exists an integer $\ell \geq 1$ such that $n$ divides $\ell(2 k-m)$ and

$$
\frac{1}{\ell}+\frac{\operatorname{gcd}(n, k)}{n}+\frac{\operatorname{gcd}(n, k-m)}{n}>1 .
$$

(2) $n=k+m$.

(3) $n=2(k-m)$ and $\operatorname{gcd}(n, k) \leq \frac{n}{2}$.

(4) $n=2 k$ and $\operatorname{gcd}(n, k-m)<\frac{n}{2}$.

\section{Asphericity}

The following theorem, due to Prishchepov, gives arithmetic conditions for the asphericity of the presentations $P(r, n, k, s, q)$.

Theorem 4. Let $P(r, n, k, s, q)$ be the symmetric presentation defined in Section 2, where either $r>2 s>0$ or $s>2 r>0$. Let $A=k-1$, $B=k-1-(r-s) q$, and suppose that one of conditions (i), (ii) and (iii) holds:

(i) $n$ does not divide any of $3 A, 4 A, 5 A, 2 B, B \pm A, B \pm 2 A, B+3 A$, $2 B+A$.

(ii) $n$ does not divide any of $3 B, 4 B, 5 B, 2 A, A \pm B, A \pm 2 B, A+3 B$, $2 A+B$.

(iii) $n$ does not divide any of $2 A, 3 A, 2 B, 3 B, A \pm B, 2 B+A, 2 A+B$.

Then the presentation $P(r, n, k, s, q)$ is aspherical. In this case, the group defined by $P(r, n, k, s, q)$ is torsion-free and infinite.

We now give a new proof of Theorem 4 by using the concept of relative presentation. We shall proceed as follows. Extending a symmetrically presented group by a finite cyclic group which cyclically permutes the set of generators and the set of relators, one obtains a group defined by a one-relator relative presentation over the finite cyclic group in question. The theory of aspherical relative group presentations, as developed by Bogley and Pride [2], applies to this set-up, there being an equivalence between relative asphericity of the relative presentation and asphericity of the original symmetric presentation. Let $\theta$ denote the automorphism of $P(r, n, k, s, q)$ which permutes cyclically the generators, i.e., $\theta\left(x_{i}\right)=x_{i+1}$ (subscripts $\left.\bmod n\right)$. Let us consider the split extension of $P(r, n, k, s, q)$ by $\mathbb{Z}_{n}=\left\langle\theta: \theta^{n}=1\right\rangle$. If we substitute relations $\theta^{-i} x_{1} \theta^{i}=x_{i+1}$ into those of $P(r, n, k, s, q)$ and set $y^{-1}=x_{1} \theta^{-q}$, 
then the split extension is generated by $\theta$ and $y$ and has a presentation

$$
Q(r, n, k, s, q)=\left\langle\theta, y: \theta^{n}=1, \quad y^{s} \theta^{k-1}=\theta^{k-1-(r-s) q} y^{r}\right\rangle .
$$

We can regard $Q(r, n, k, s, q)$ as a relative presentation in the sense of Bogley and Pride, that is,

$$
Q(r, n, k, s, q)=\left\langle H, y: y^{s} \theta^{A}=\theta^{B} y^{r}\right\rangle
$$

where $H=\left\langle\theta: \theta^{n}=1\right\rangle, A=k-1$ and $B=k-1-(r-s) q$.

Lemma 5. If the relative presentation $Q(r, n, k, s, q)$ is aspherical, then the ordinary presentation $P(r, n, k, s, q)$ is aspherical.

Proof: Let $\mathbf{P}$ be a spherical picture over the ordinary presentation $P(r, n, k, s, q)$. Then $\mathbf{P}$ contains discs $\Delta_{i}$ corresponding to relations

$$
\left(\prod_{j=1}^{r} x_{i+(j-1) q}\right)\left(\prod_{j=1}^{s} x_{i+k-1+(s-j) q}^{-1}\right)=1
$$

as shown in Figure 1.

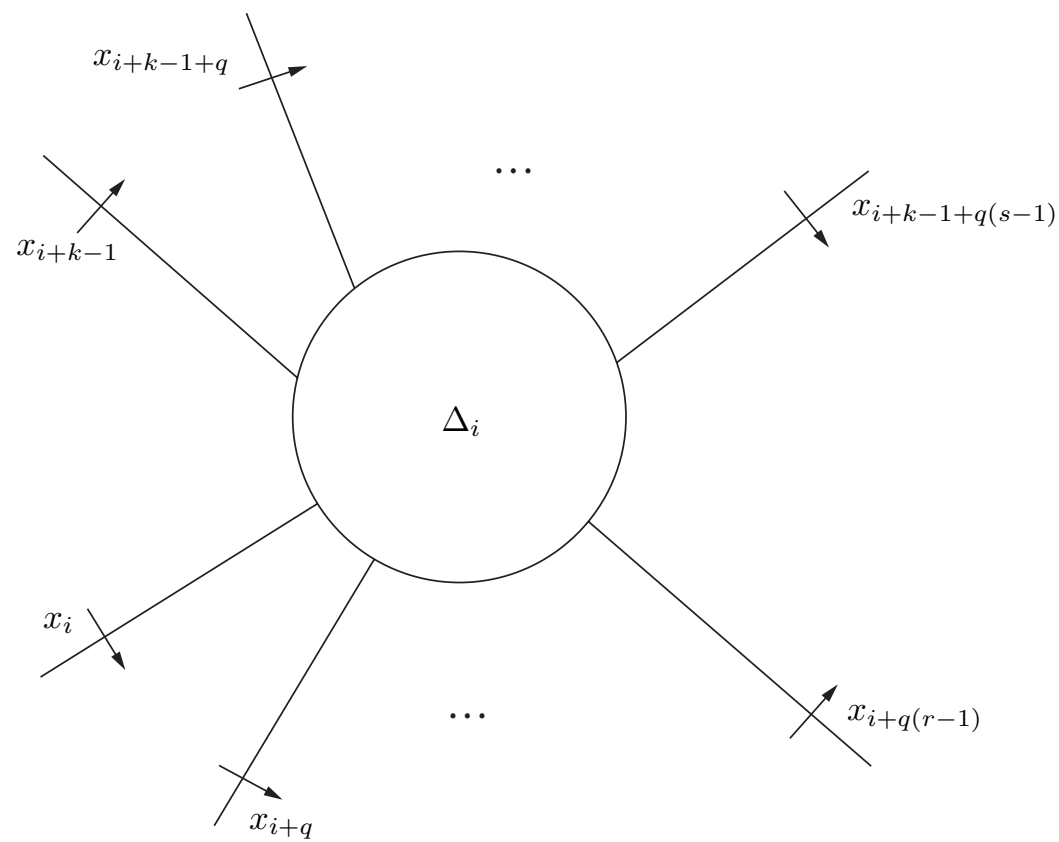

Figure 1. An inner disc in a spherical picture $\mathbf{P}$ over $P(r, n, k, s, q)$. 
Here we have no labels at the corners since we regard the ordinary presentation $P(r, n, k, s, q)$ as a relative presentation with $H=1$. Let us replace each inner disc $\Delta_{i}$ by a picture $\Sigma_{i}$ over $Q(r, n, k, s, q)$ considered as an ordinary presentation (see Figure 2). Here we have replaced arcs labelled by $x_{i+j q}$ (and similarly for $x_{i+k-1+j q}^{-1}$ ) by sequences of arcs using relations

$$
x_{i+j q}=\theta^{-(i+j q-1)} x_{1} \theta^{i+j q-1}=\theta^{-(i+j q-1)} y^{-1} \theta^{i+(j+1) q-1} .
$$

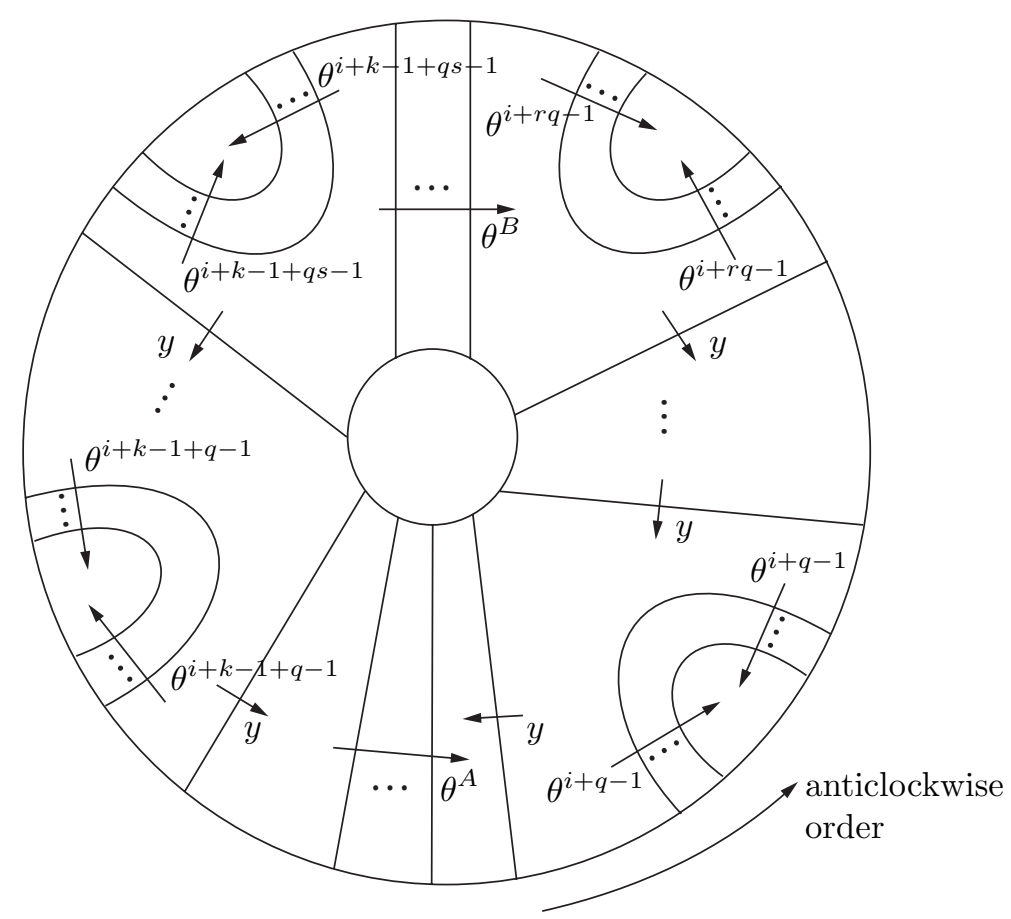

Figure 2. The picture $\Sigma_{i}$ over the ordinary presentation $Q(r, n, k, s, q)$.

Along the boundary of $\Sigma_{i}$ we get the relation

$$
\begin{aligned}
\left(\prod_{j=1}^{r} y^{-1} \theta^{i+j q-1} \theta^{-(i+j q-1)}\right) \theta^{-B} & \\
\left(\prod_{j=1}^{s} \theta^{i+k-1+(s+1-j) q-1} \theta^{-(i+k-1+(s+1-j) q-1)} y\right) \theta^{A} & =1
\end{aligned}
$$


which is equivalent to the $i$-th relation of $P(r, n, k, s, q)$. Along the boundary of the interior disc in $\Sigma_{i}$ we get the relation

$$
y^{-r} \theta^{-B} y^{s} \theta^{A}=1
$$

which is a relation of $Q(r, n, k, s, q)$. The arcs of $\Sigma_{i}$ having both ends on $\partial \Sigma_{i}$ can be made into floating circles. These circles can be removed from the resulting picture. Furthermore, we will replace all other arcs with $\theta$-labels by corner labels on the disc as shown in Figure 3 . We get again the relation $y^{-r} \theta^{-B} y^{s} \theta^{A}=1$.

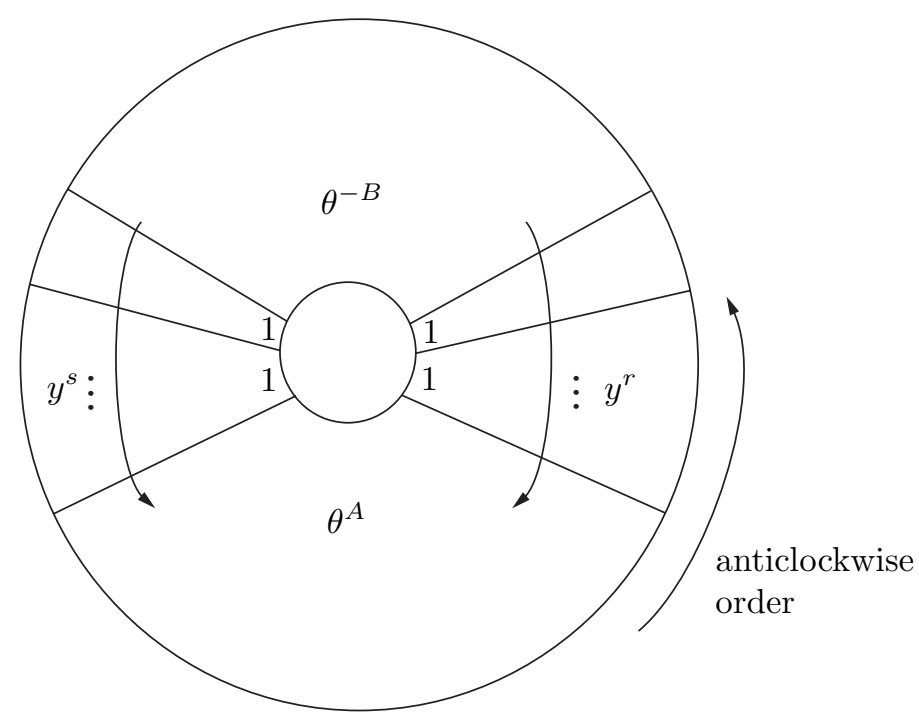

Figure 3. A picture $\mathbf{Q}$ over the relative presentation $Q(r, n, k, s, q)$.

Repeating the same construction for each disc $\Delta_{i}$ of $\mathbf{P}$ yields a picture $\mathbf{Q}$ over the relative presentation $Q(r, n, k, s, q)$. By the assumption of asphericity for $Q(r, n, k, s, q)$, the picture $\mathbf{Q}$ must contain a dipole, i.e., a pair of opposite oriented discs connected by an arc which define pairwise inverse words (see Figure 4). 

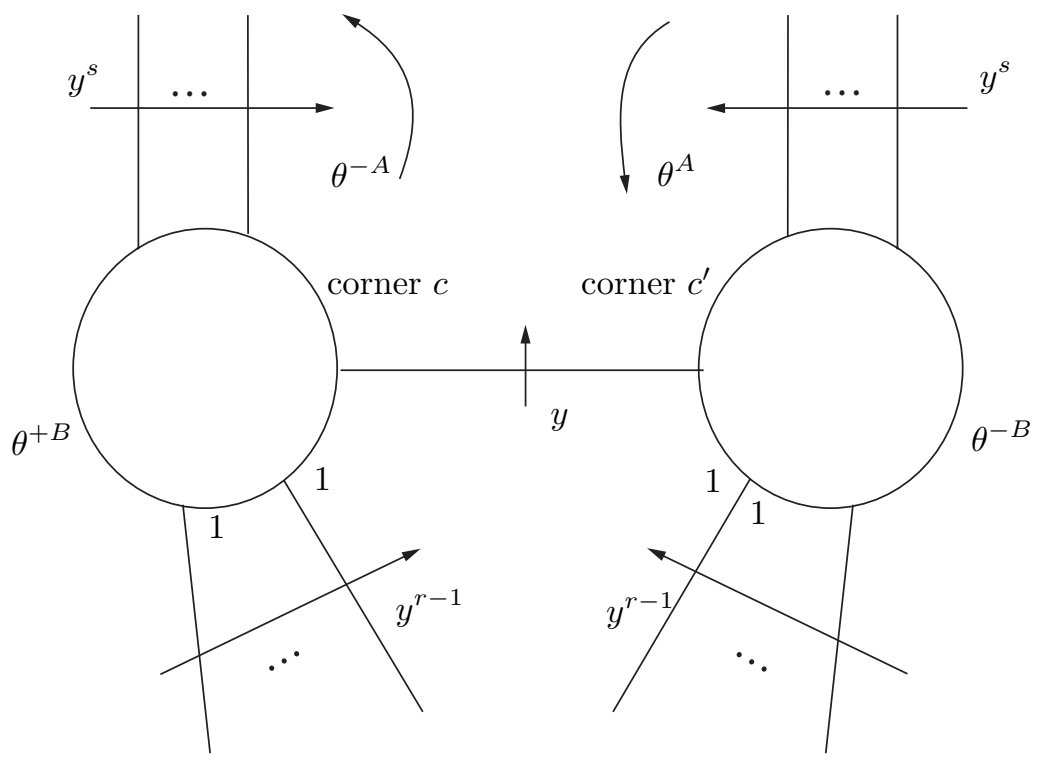

Figure 4. A dipole in the picture $\mathbf{Q}$ over the relative presentation $Q(r, n, k, s, q)$.

It is easy to see that any such dipole in $\mathbf{Q}$ arises from a pair of identical but oppositely oriented discs in $\mathbf{P}$ connected by an arc with label $x_{i}$ for some $i$. Moreover, two bridge moves in $\mathbf{P}$ produce a cancelling pair of discs. This means that if $\mathbf{Q}$ has a pair of cancelling discs, then $\mathbf{P}$ has a pair of cancelling discs, too. Thus, the initial picture $\mathbf{P}$ must contain a dipole. Therefore, any nonempty spherical picture over $P(r, n, k, s, q)$ is equivalent to one having two fewer discs, hence this presentation is aspherical by induction.

To study the asphericity of the relative presentation

$$
Q(r, n, k, s, q)=\left\langle H, y: y^{s} \theta^{A}=\theta^{B} y^{r}\right\rangle
$$

where $H=\left\langle\theta: \theta^{n}=1\right\rangle$, we use the following algebraic criterion, due to Prishchepov, which is stated here in terms of relative presentations. 
Theorem 6. Let $G$ be a group defined by the relative presentation

$$
\left\langle H, y: y^{s} d=a y^{r}\right\rangle
$$

for some group $H$, where either $r>2 s>0$ or $s>2 r>0$. Then $G$ is aspherical if one of conditions (i), (ii) and (iii) holds in $H$ :

$$
\begin{aligned}
& \text { (i) }\left\{\begin{array}{l}
a \text { is of order at least } 3 \\
d \text { is of order at least } 6 \\
a d^{ \pm 1} \neq 1, a d^{ \pm 2} \neq 1, a d^{3} \neq 1, a^{2} d \neq 1
\end{array}\right. \\
& \text { (ii) }\left\{\begin{array}{l}
a \text { is of order at least } 6 \\
d \text { is of order at least } 3 \\
d a^{ \pm 1} \neq 1, d a^{ \pm 2} \neq 1, d a^{3} \neq 1, d^{2} a \neq 1
\end{array}\right. \\
& \text { (iii) }\left\{\begin{array}{l}
a \text { is of order at least } 4 \\
d \text { is of order at least } 4 \\
d a^{ \pm 1} \neq 1, d a^{2} \neq 1, d^{2} a \neq 1 .
\end{array}\right.
\end{aligned}
$$

In these cases, $y$ is of infinite order in $G$ and does not commute with any non-identity element of $H$.

We now apply Theorem 6 to our case where $a=\theta^{B}, d=\theta^{A}, A=k-1$ and $B=k-1-(r-s) q$. One can directly verify that cases (i), (ii) and (iii) of Theorem 6 produce the corresponding ones in the statement of Theorem 4. Finally, recall that the group presented by $Q(r, n, k, s, q)$ is infinite if and only if the group presented by $P(r, n, k, s, q)$ is infinite.

\section{Topological results}

Throughout the section let $G=G(r, n, k, s, q)$ denote the group defined by the symmetric presentation $P=P(r, n, k, s, q)$, and let $K=$ $K(P)$ be the canonical 2-complex associated to $P$.

The following results were proved in [8].

Theorem 7. Suppose that $r+s(\geq 3)$ is odd, and $n(\geq 3)$ is odd and coprime with $2(k-1)+q(s-r)$. Then the Prishchepov group $G(r, n, k, s, q)$ cannot be the fundamental group of a hyperbolic 3-orbifold (in particular, a closed 3-manifold) of finite volume. 
Theorem 8. The abelianization of the group $G(r, n, k, s, q)$ is infinite if and only if one of the following conditions holds:

(i) $s=r$,

(ii) there exists $m \in \mathbb{Z}, m>1, m \backslash n, m$ does not divide qs, with $q s \equiv q r(\bmod m)$, and $k \equiv 1(\bmod m)$,

(iii) there exists $m \in \mathbb{Z}, m>1, m \backslash n, m$ does not divide qs, with $q s \equiv-q r(\bmod m)$, and $k+q s \equiv 1+m / 2(\bmod m), m$ even.

In the finite case, the natural $H N N$ extension of $G(r, n, k, s, q)$ is a 3-knot group.

Recall that a hyperbolic 3 -orbifold is the quotient space $\mathbb{H}^{3} / \Gamma$, where $\mathbb{H}^{3}$ is the hyperbolic 3 -space and $\Gamma$ is a discrete group of isometries of $\mathbb{H}^{3}$ (in particular, if $\Gamma$ is torsion-free, then we get the notion of $h y$ perbolic 3-manifold). A 3-knot is a locally flat topological embedding of $\mathbb{S}^{3}$ into $\mathbb{S}^{5}$.

Proposition 9. Let $P=P(r, n, k, s, q)$ be orientable and satisfy one of the conditions in the statement of Theorem 4. Then the Prishchepov group $G=G(r, n, k, s, q)$ cannot be the fundamental group of a closed connected orientable 3-manifold.

Proof: Suppose, on the contrary, that $M^{3}$ is a closed connected orientable 3 -manifold such that $\pi_{1}(M) \cong G$. By Theorem 1 the canonical 2-complex $K=K(P)$ is aspherical, i.e., $K=K(G, 1)$. Since $G$ is torsion-free, the prime factors of $M$ are either aspherical or isomorphic to $\mathbb{S}^{1} \times \mathbb{S}^{2}$ (or counterexamples to the Poincaré conjecture). So if $G$ has $k$ freely indecomposable free factors, then we have

$$
1=\chi(K)=\chi(G)=\chi(M)+1-k \leq 0
$$

which is a contradiction.

Theorem 10. Let $G=G(r, n, k, s, q)$ be as in Proposition 9. Then there exists a smooth closed orientable spin 4-manifold $M^{4}$ such that:

(1) $\chi(M)=2, \pi_{1}(M) \cong G, \pi_{2}(M) \cong \overline{\operatorname{Ext}}_{\Lambda}^{2}(\mathbb{Z}, \Lambda) \cong \bar{H}^{2}(G ; \Lambda)$, where $\Lambda=\mathbb{Z}[G]$ is the integral group ring of $G$ (for a right $\Lambda$-module $A$, the symbol $\bar{A}$ represents the associated left $\Lambda$-module induced by the canonical anti-automorphism $-: \Lambda \rightarrow \Lambda$ sending $g$ to $g^{-1}$ ); 
(2) $M$ bounds a smooth compact 5-manifold $N^{5} \subset \mathbb{R}^{5}$ such that $N \simeq$ $K(G, 1)$;

(3) The first $k$-invariant and the signature of $M$ vanish;

(4) The integral homology of the universal cover $\widetilde{M}$ of $M$ is $H_{1}(\widetilde{M}) \cong$ $H_{4}(\widetilde{M}) \cong 0, H_{2}(\widetilde{M}) \cong \bar{H}^{2}(G ; \Lambda)$, and $H_{3}(\widetilde{M}) \cong Z^{e(G)-1}$, where $e(G)$ is the number of ends of $G$.

If $e(G)>1$, then $G$ is a nontrivial free product. If $e(G)=1$ and $H^{2}(G ; \Lambda)$ is finitely generated, then $\widetilde{M}$ is homotopy equivalent to $\mathbb{S}^{2}$ (hence $\pi_{2}(M) \cong \bar{H}^{2}(G ; \Lambda) \cong \mathbb{Z}$ and $\left.H^{1}(G ; \Lambda) \cong 0\right)$. If the abelianization $G^{\mathrm{ab}}$ of $G$ is finite (see Theorem 8), then $M^{4}$ is a rational homology 4-sphere, and there is an epimorphism from $\pi_{2}(M)$ onto $H_{2}(M ; \mathbb{Z}) \cong G^{\mathrm{ab}}$. If further $H^{2}(G ; \Lambda)$ is finitely generated, then $G^{\text {ab }}$ is finite cyclic (possibly null).

Proof: Embed the canonical 2-complex $K=K(P)$ into $\mathbb{R}^{5}$, and define $M^{4}$ to be the boundary of a regular neighborhood $N^{5}$ of $K$ in $\mathbb{R}^{5}$. Since $N$ collapses onto $K$, we have $N \simeq K(G, 1)$ and $\chi(N)=1$. One easily checks $\chi(M)=2 \chi(N)=2$. By [13] and Corollary 5.2, p. 116, of [15] there are isomorphisms $\pi_{2}(M) \cong \overline{\operatorname{Ext}}_{\Lambda}^{2}(\mathbb{Z}, \Lambda) \cong \bar{H}^{2}(G ; \Lambda)$. Since $G$ has cohomological dimension $\leq 2$, we have $H^{3}\left(G ; \pi_{2}(M)\right) \cong 0$, hence the first $k$-invariant of $M$ vanishes. Furthermore, $M$ is spin and its signature is zero as $M$ embeds in $\mathbb{R}^{5}$. The integral homology of $\widetilde{M}$ is given by $H_{1}(\widetilde{M}) \cong 0, H_{2}(\widetilde{M}) \cong \pi_{2}(M), H_{3}(\widetilde{M})=H_{3}(M ; \Lambda) \cong \bar{H}^{1}(M ; \Lambda) \cong$ $\bar{H}^{1}(G ; \Lambda) \cong \mathbb{Z}^{e(G)-1}$, and $H_{4}(\widetilde{M}) \cong 0$ (recall that $G$ is infinite). If the group $G$ has more than one end, then it is isomorphic to a nontrivial generalized free product with amalgamation $U *_{W} V$ or an HNN extension $U *_{W} \phi$, where $W$ is finite and $U \neq W \neq V$ (see for example [12, p. 11]). Since $G$ is torsion free, we must have $W=1$, hence $G$ is isomorphic to either $U * V$ or $U * \mathbb{Z}$, where $U, V \neq 1$. Thus $G$ is a nontrivial free product.

If $e(G)=1$ and $H^{2}(G ; \Lambda)$ is finitely generated, then $H_{*}(\widetilde{M} ; \mathbb{Z})$ is finitely generated. By Corollary C, p. 23, of [12], $M$ is either aspherical or $\widetilde{M}$ is homotopy equivalent to $\mathbb{S}^{2}$ or $\mathbb{S}^{3}$ or $\pi_{1}(M)$ is finite. The first case cannot occur since otherwise $\chi(M)=\chi(G)=1$ contradicts $\chi(M)=2$. By Theorem 10 (i), p. 23, of [12], $\widetilde{M}$ is homotopy equivalent to $\mathbb{S}^{3}$ if and only if $e(G)=2$ and $\chi(M)=0$. Thus it remains only the case $\widetilde{M} \simeq \mathbb{S}^{2}$, hence $\pi_{2}(M) \cong \bar{H}^{2}(G ; \Lambda) \cong \mathbb{Z}$ and $H^{1}(G ; \Lambda) \cong 0$. 
If the abelianization $G^{\mathrm{ab}}$ of $G$ is finite, then $\chi(M)=2=2-2 \beta_{1}(M)+$ $\beta_{2}(M)=2+\beta_{2}(M)$ implies that $\beta_{2}(M)=0$. Thus $M$ is a rational homology 4 -sphere. Since $G^{\mathrm{ab}}$ is finite, we have also $\beta_{1}(K)=0$. Then $\chi(K)=1=1+\beta_{2}(K)$ gives $\beta_{2}(K)=0$, hence $H_{2}(K ; \mathbb{Z}) \cong 0$. It follows that $H_{2}(G ; \mathbb{Z}) \cong 0$ by the Hopf formula. In fact, this formula states that the number of generators of $H_{2}(G ; \mathbb{Z})$ is $\alpha-\beta+\gamma$, where $\beta$ is the number of generators and $\alpha$ the number of relations of $G$ while $\gamma$ is the rank of $H_{1}(G)=G^{\text {ab }}$ (see for example [3, p. 46]). In our case, we have $\alpha=\beta=n$ and $\gamma=0$. Let us consider the terms of low degree of the spectral sequence of the universal cover of $M$, that is, the exact sequence

$$
\cdots \longrightarrow H_{2}(\widetilde{M}) \cong \pi_{2}(M) \longrightarrow H_{2}(M) \longrightarrow H_{2}(G) \cong 0 .
$$

Since $H_{2}(M) \cong H^{2}(M) \cong F H_{2}(M) \oplus T H_{1}(M) \cong G^{\text {ab }}$, we have an epimorphism from $\pi_{2}(M) \cong \bar{H}^{2}(G ; \Lambda)$ onto $G^{\text {ab }}$. Farrell [9] has shown that if $G$ is finitely presentable and has an element of infinite order, then $H^{2}(G ; \Lambda)$ is either $0, \mathbb{Z}$, or is not finitely generated. So, if $H^{2}(G ; \Lambda)$ is finitely generated, then $G^{\text {ab }}$ is finite cyclic (possibly null).

The following arises in a natural way:

Open problem. Compute $H^{2}(G ; \Lambda)$ and determine the ends of the Prishchepov group $G=G(r, n, k, s, q)$ for arbitrary values of the parameters.

Acknowledgements. Work performed under the auspices of the G.N.S.A.G.A. of the C.N.R. (National Research Council) of Italy and partially supported by the Ministero dell'Istruzione, Università e Ricerca of Italy within the project Proprietà Geometriche delle Varietà Reali e Complesse, and by a Research grant of the University of Modena and Reggio Emilia.

\section{References}

[1] V. G. Bardakov and A. Yu. Vesnin, On a generalization of Fibonacci groups, (Russian), Algebra Logika 42(2) (2003), 131-160; translation in: Algebra Logic 42(2) (2003), 73-91.

[2] W. A. Bogley And S. J. Pride, Aspherical relative presentations, Proc. Edinburgh Math. Soc. (2) 35(1) (1992), 1-39.

[3] K. S. Brown, "Cohomology of Groups", Graduate Texts in Mathematics 87, Springer-Verlag, New York-Berlin, 1982.

[4] C. M. Campbell and E. F. Robertson, On a class of finitely presented groups of Fibonacci type, J. London Math. Soc. (2) 11(2) (1975), 249-255. 
[5] A. Cavicchioli, Neuwirth manifolds and colourings of graphs, Aequationes Math. 44(2-3) (1992), 168-187.

[6] A. Cavicchioli, F. Hegenbarth And A. C. Kim, A geometric study of Sieradski groups, Algebra Colloq. 5(2) (1998), 203-217.

[7] A. Cavicchioli, F. Hegenbarth and D. Repovš, On manifold spines and cyclic presentations of groups, in: "Knot Theory" (Warsaw, 1995), Banach Center Publ. 42, Polish Acad. Sci., Warsaw, 1998, pp. 49-56.

[8] A. Cavicchioli, D. Repovš and F. Spaggiari, Topological properties of cyclically presented groups, J. Knot Theory Ramifications 12(2) (2003), 243-268.

[9] F. T. FARrell, The second cohomology group of $G$ with $\mathbb{Z}_{2}[G]$ coefficients, Topology 13 (1974), 313-326.

[10] N. D. Gilbert AND J. Howie, LOG groups and cyclically presented groups, J. Algebra 174(1) (1995), 118-131.

[11] H. M. Hilden, M. T. Lozano and J. M. MontesinosAmilibia, The arithmeticity of the figure eight knot orbifolds, in: "Topology '90" (Columbus, OH, 1990), Ohio State Univ. Math. Res. Inst. Publ. 1, de Gruyter, Berlin, 1992, pp. 169-183.

[12] J. A. Hillman, "The algebraic characterization of geometric 4-manifolds", London Mathematical Society Lecture Note Series 198, Cambridge University Press, Cambridge, 1994.

[13] J. A. Hillman, Minimal 4-manifolds for groups of cohomological dimension 2, Proc. Edinburgh Math. Soc. (2) 37(3) (1994), 455-461.

[14] D. L. Johnson, J. W. Wamsley and D. Wright, The Fibonacci groups, Proc. London Math. Soc. (3) 29 (1974), 577-592.

[15] S. Mac Lane, "Homology", Die Grundlehren der mathematischen Wissenschaften 114, Academic Press, Inc., Publishers, New York; Springer-Verlag, Berlin-Göttingen-Heidelberg, 1963.

[16] R. W. K. Odoni, Some Diophantine problems arising from the theory of cyclically-presented groups, Glasg. Math. J. 41(2) (1999), $157-165$.

[17] M. I. Prishchepov, Asphericity, atoricity and symmetrically presented groups, Comm. Algebra 23(13) (1995), 5095-5117.

[18] A. Szczepański And A. Yu. Vesnin, On generalized Fibonacci groups with an odd number of generators, Comm. Algebra 28(2) (2000), 959-965. 
Department of Mathematics

University of Modena and Reggio E.

Via Campi 213/B

41100 Modena

Italy

E-mail address: spaggiari.fulvia@unimo.it

Rebut el 8 de març de 2005. 